\title{
The FPU problem as a statistical-mechanical counterpart of the KAM problem, and its relevance for the foundations of physics
}

\author{
A. Carati* L.Galgani*, A. Maiocchi, F. Gangemi, R. Gangemi ${ }^{\dagger}$
}

December 15, 2018

\begin{abstract}
We give a review of the Fermi-Pasta-Ulam (FPU) problem from the perspective of its possible impact on the foundations of physics, concerning the relations between classical and quantum mechanics. In a first part we point out that the problem should be looked upon in a wide sense (whether the equilibrium Gibbs measure is attained) rather than in the original restricted sense (whether energy equipartition is attained). A second part is devoted to some very recent results of ours for an FPU-like model of an ionic crystal, which has such a realistic character as to reproduce in an impressively good way the experimental infrared spectra. Since the existence of sharp spectral lines is usually considered to be a characteristic quantum phenomenon, even unconceivable in a classical frame, this fact seems to support a thesis suggested by the original FPU result. Namely, that the relations between classical and quantum mechanics are much subtler than usually believed, and should perhaps be reconsidered under some new light.
\end{abstract}

Keywords: FPU problem, foundations of statistical mechanics, relations between classical and quantum physics.

MSC classification: $82 \mathrm{C} 03$.

\section{Introduction}

The Fermi-Pasta-Ulam (or FPU) problem was introduced in the year 1954, the same year the Kolmogorov-Arnold-Moser (or KAM) theorem was first formulated and proved. Such a problem gave rise to a lot of researches, as is witnessed for example by two collections of works reported in a special issue of the journal Chaos [1] and in a book edited by Gallavotti [2]. In the present review, however,

\footnotetext{
*Department of Mathematics, Università degli Studi di Milano -E-mail: andrea.carati@unimi.it

${ }^{\dagger}$ DMMT, Università di Brescia, Viale Europa 11, I-25123 Brescia, Italy
} 
we limit ourselves to consider the features which are relevant for a question concerning the foundations of physics that was raised already in the year 1966 by Chirikov and his group, along the Fermi original motivation. The question is whether the results obtained on the FPU problem shed some new light on the relations between classical and quantum mechanics, perhaps along the lines of the "classical program" envisaged by Einstein (see [3], pages 664-687, and [4]). Thus, no attempt is made to address the many other facets the FPU problem may present. Surveys of the many contributions given, can be found for example in the two collections of works [1] [2] mentioned above.

It will be shown that the results obtained up to now don't yet allow one to decide the question of the relations between classical and quantum mechanics, although strong indications exist that such relations be much subtler that usually believed, and should perhaps be reconsidered under some new light. The strongest indication will be illustrated in the last part of the present review, which is devoted to recent results of ours on an FPU-like model of an ionic crystal, having such a realistic character as to reproduce in an impressively good way, in the case of Lithum Fluoride, the experimental infrared spectra, a characteristic quantum phenomenon, indeed. It might be appropriate to recall here that, in Born's words ([5], Chapter V, Section 1) "sharp spectral lines ... [are] ... phenomena which from the classical standpoint are perfectly unintelligible".

The last part actually constitutes the main originality of the present review, with respect to two previous ones written by the two first authors, together with Giorgilli [6] and with Benettin and Giorgilli [7] respectively. It will be seen however that also the first part of the review, which partially superposes the previous ones, contains some relevant indications, related to certain features of the FPU problem that did emerge in the meantime.

This paper pays a tribute to Jürgen Moser, to whom the present issue of the journal Regular and Chaotic Dynamics is dedicated. In the years seventies he invited to Oberwolfach the older of the present authors, together with Benettin and Giorgilli, for a conference devoted to mathematical features of dynamical systems theory. But Moser was eager to discuss also the physical relevance of the FPU problem, and thus the present paper can also be seen as an ideal continuation of those discussions. In such a spirit, the style will be rather informal.

In the next section we recall how the FPU problem originated from the same mathematical frame as KAM theory, but with a physical motivation. In the two following sections we will give, in a concise but hopefully clear way, a review of the results obtained up to now, collecting them in two phases, which deal with what we call the strict-sense problem (section 3) and the wide-sense problem (section 4) respectively. Finally, in section 5 we summarize some very recent results of ours on a realistic FPU-like model of an ionic crystal, which provide the strongest evidence for the necessity of reconsidering the relations between classical and quantum mechanics. Actually, one should rather speak of relations between classical physics and quantum physics, inasmuch as the electromagnetic field emerges as playing an essential role in such relations. Some comments are finally given in the conclusive section. 


\section{Mathematical and physical origins of the FPU problem}

Let us start recalling how the two problems (FPU and KAM) did originate within the same mathematical frame, namely the dynamics of slightly perturbed integrable Hamiltonian systems. In both cases the aim was to overcome, from two complementary approaches, the difficulties raised by a celebrated apparently paradoxical (or at least disconcerting) result of Poincaré concerning the existence of integrals of motion. [8]. Namely, one considers an integrable Hamiltonian system, which thus presents a number of integrals of motion equal to the number $N$ of degrees of freedom, and subjects it to a Hamiltonian perturbation. The result then is that "in general" the perturbed system admits no integral of motion apart from energy, however small (this is the disconcerting point) the perturbation may be.

Now, in such an apparently paradoxical situation continuity was recovered in a measure-theoretic sense, and indeed in an astonishing way, through KAM theorem. Namely, single $N$ dimensional invariant tori were proven to exist, and the corresponding "strange" set of invariant tori was proven to have full measure in the limit of vanishing perturbation. These facts about KAM theory are well known. We now recall how in the FPU problem one is faced with an analogous, apparently paradoxical, situation of discontinuity, which has a deep impact on the relations between classical and quantum mechanics.

The FPU problem [9] shows up if one looks at the same mathematical frame from the point of view of statistical mechanics. In a nutshell, the heart of the problem is as follows. One considers a particular integrable system, namely, a system of $N$ harmonic oscillators of different frequencies, with a nonlinear coupling. Actually, one considers a fundamental model of physics, namely, a chain of particles mimicking a one-dimensional crystal, in which case the harmonic oscillators are nothing but the normal modes of the linearized model, and the role of the coupling parameter is played by energy itself (because the perturbation is just the nonlinearity, which vanishes for vanishing energy).

With respect to the general mathematical frame described above one has to add that, for the aims of statistical mechanics, the absence of further integrals apart from total energy might be expected to entail that, at equilibrium, the statistical properties of the system are well described by the Gibbs measure. Now, the Gibbs statistics entails equipartition (i.e., equal share) of energy among the modes, in sharp contrast with the unperturbed case in which, due to the presence of $N$ integrals, energy always remains distributed among the modes according to the initial conditions, without any restriction. So we have here an analogue of the discontinuity recalled above in the case of KAM theorem. The relevant point is that the equipartition "theorem" is a result of fundamental importance for physics, since its empirical failure (at high frequencies for the black-body case, and at low energies for the specific heat of solids) is the fact that triggered the very birth of quantum mechanics in the year 1900 with Planck and in the year 1907 with Einstein, when Planck's law was substituted for the 
"classical" equipartition law.

Thus one has to understand whether really energy equipartition among the normal modes at low energies is the prediction of classical statistical mechanics. This problem was tackled by FPU in the most natural way, namely, by checking whether in their model (to be recalled below) energy equipartition is attained, if one starts from initial conditions with only a few modes excited. This might be called the strict-sense FPU problem. More in general, one may ask whether the Gibbs distribution is attained, which might be called the wide-sense FPU problem.

At first sight a sharp alternative seems to occur. If at low energy no further integral exists, or more in general if no dynamical obstacle to ergodicity occurs, then at equilibrium classical statistical mechanics might be expected to be applicable, no matter how small the perturbation (the energy) may be. So equipartition obtains at all energies (this is the paradoxical discontinuous face of the alternative), and consequently classical mechanics itself qualitatively fails, and the necessity of quantization is mathematically proven. If instead some more complicated situation is met by which some sort of continuity is restored, then the relations between classical and quantum mechanics should be understood in some new, subtler way.

We are convinced that these are the questions Fermi had in mind when, together with Pasta and Ulam, he tackled the problem through numerical computations, as soon as computers started to be available for scientific research, after the war. Indeed already in his youth he had even given a proof of a stronger version of the Poincaré theorem [10], not because of any purely mathematical interest of his in ergodic theory, but because of his interests in the foundations of physics. ${ }^{1}$

The results found by FPU, apparently in favour of nonapplicability of Gibbs methods and of the necessity of reconsidering the foundational problem, will be illustrated in a moment. The reactions of Fermi are unknown, as he unfortunately passed away before the paper was even written down by the other two authors. We have available, however, the witness of Ulam who, in a preface to the FPU work reproduced in Fermi's Collected Works, after recalling "Fermi's earlier interest in the ergodic theory" says: "The results of the calculations were interesting and quite surprising to Fermi. He expressed to me the opinion that they really constituted a little discovery in providing intimations that the prevalent beliefs in the universality of mixing and thermalization in non-linear systems may not be always satisfied". In other words, the FPU result was apparently against the common opinion that equipartition holds at all energies. Consequently it is not granted that classical mechanics fails in some obvious

\footnotetext{
${ }^{1}$ Notice that, as emphasized by his pupil Segrè in the introduction to Fermi's Collected Papers (page XXVII), Fermi had no interest for mathematics in itself, being only interested in the contribution mathematics can give to Physics. Also of interest is a conversation the senior author had once with Segrè in Berkeley, concerning Fermi's attitude towards quantum mechanics. Segrè said that Fermi, due to his well known reserved character, didn't like to stand public discussions about the foundations of quantum mechanics, but he trusted his friends he was malcontent with the standard interpretation of the new mechanics.
} 
way. This is the "little discovery" alluded to by Fermi, and sometimes called "the FPU paradox".

\section{First phase. The strict-sense FPU problem and the debate about the chaoticity threshold}

\subsection{The original FPU result}

Let us start recalling the FPU model. One considers a system of $N+2$ particles on a line, with the positions $x_{0}, x_{N+1}$ of the extreme particles kept fixed, while the positions $x_{1}, x_{2}, \ldots x_{N}$ of the remaining ones are the free coordinates. One has certain nearest-neighbour nonlinear interactions, as if due to springs connecting adjacent points. One thus has, in suitable units, a Hamiltonian of the type

$$
H=\sum_{i=1}^{N} \frac{p_{i}^{2}}{2}+\sum_{i=1}^{N+1} V\left(x_{i}-x_{i-1}\right),
$$

where the potential energy of each spring is taken of the form

$$
V(y)=\frac{y^{2}}{2}+\alpha \frac{y^{3}}{3}+\beta \frac{y^{4}}{4}
$$

with two parameters $\alpha, \beta$. For example, in the case $\beta=0$ the equations of motion have the form (for $i=1, \ldots, N$ )

$$
\ddot{x}_{i}=x_{i+1}+x_{i-1}-2 x_{i}+\alpha\left[\left(x_{i+1}-x_{i}\right)^{2}-\left(x_{i}-x_{i-1}\right)^{2}\right] .
$$

\section{Passage to the normal oscillation modes}

Since the year 1759 it is known that in the linearized case $(\alpha=\beta=0)$, passing to a suitable set of coordinates $a_{1}, \ldots a_{N}$, the system is equivalent to a system of independent harmonic oscillators. One has thus a Hamiltonian

$$
H_{0}=\sum_{j=1}^{N} \frac{1}{2}\left(\dot{a}_{j}^{2}+\omega_{j}^{2} a_{j}^{2}\right)
$$

with equations of motion

$$
\ddot{a}_{j}+\omega_{j}^{2} a_{j}=0
$$

and certain frequencies

$$
\omega_{j}=2 \sin \frac{\pi j}{2(N+1)} .
$$

The (linear) change of variables that makes the job is

$$
a_{j}=\sum_{k} x_{k} \sin \frac{\pi k j}{N+1} .
$$


For nonvanishing values of $\alpha$ and/or $\beta$ the equations of motions in terms of the mode amplitudes $a_{j}$ are quite complicated, because each mode in general interacts with all the other ones, and in general one has equations of the form

$$
\ddot{a}_{j}=-\omega_{j}^{2} a_{j}+\text { higher order terms . }
$$

By numerical solutions of the equations of motion, one thus obtains the positions and velocities of the particles, and through the change of variables (2) from time to time one also determines the mode amplitudes $a_{j}$ and the corresponding velocities, and thus also the mode energies

$$
E_{j}=\frac{1}{2}\left(\dot{a}_{j}^{2}+\omega_{j}^{2} a_{j}^{2}\right)
$$

\section{The FPU result}

As was already said, the aim of FPU was to check whether energy equipartition for the time averages of the energies $E_{j}$ is attained, if one starts from an exceptional initial state in which only one or a few modes are excited (usually, a packet of low-frequency modes). This is what was called above the strict-sense FPU problem. FPU reported the results of several computations for 32 or 64 particles, exhibiting figures of the instantaneous amplitudes of a selection of modes, plotted versus time. The essence of their results, however, is embodied in the last of their figures where they report, vs time $t$, the time average of a selection of modes up to time $t$, namely, the quantities $\overline{E_{j}}(t, z)$ defined by

$$
\overline{E_{j}}(t, z)=\frac{1}{t} \int_{0}^{t} E_{j}\left(\Phi^{s}(z)\right) \mathrm{d} s .
$$

where $\Phi^{s}(z)$ denotes the point in phase space evolved at time $s$ from point $z$. Indeed, time averages of significant phase-space functions are the quantities of interest for ergodic theory.

An analogue of their last figure is reported here in Figure 1, that we obtained (for $N=63$ and $\alpha=\beta=1 / 4$ ) with only the lowest-frequency mode initially excited (with vanishing velocity), at total energy $E=0.25$. Notice that, when looking at figures of this type, one should take into account that a natural time unit for the FPU system is the period $T_{N}=2 \pi / \omega_{N}$ of the highest-frequency mode, namely, $T_{N}=\pi$.

The figure exhibits the result that so much impressed Fermi. Namely, up to the available integration time not only energy equipartition is not attained, but furthermore the system apparently relaxed to some stationary state, since the time averaged energies seem to have attained some limits. In connection with the general problem previously discussed, one has the impression that some kind of continuity is exhibited here with respect to the case of vanishing perturbation. Indeed, in the latter case the energy of each mode is an integral of motion. Then, apparently, a small perturbation has the effect that the mode energies are no more integrals of motion, but the energy share among the modes occurs in such a way, that the final state is reminiscent of the initial condition, since the "final energies" are a decreasing function of frequency. 


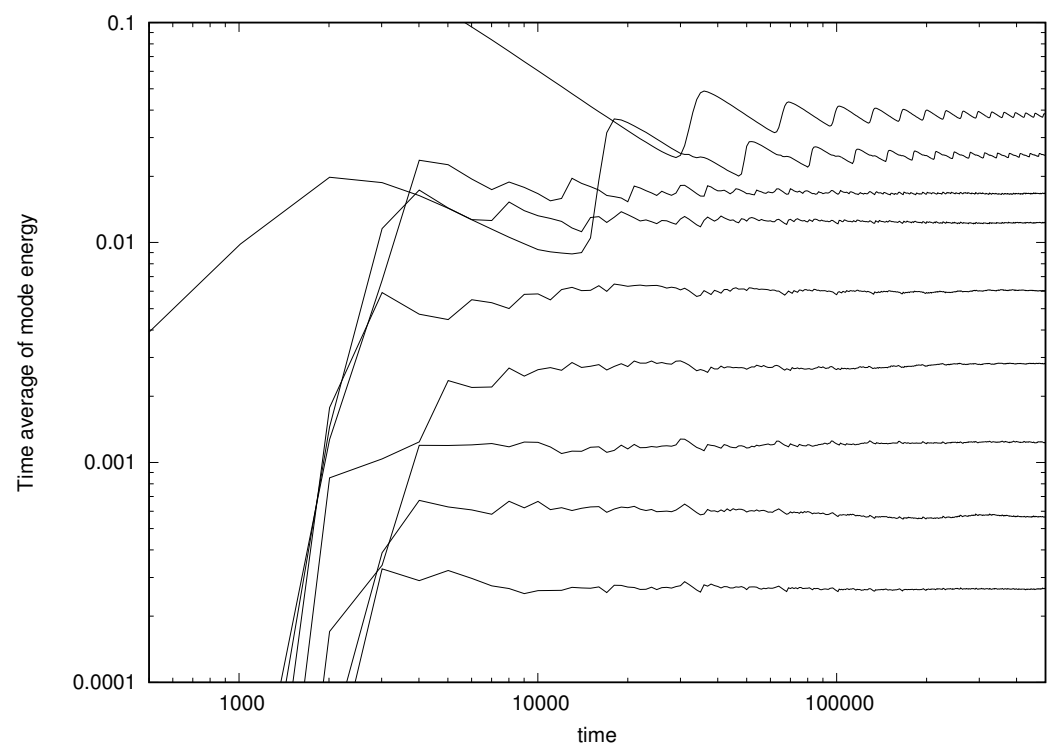

Figure 1: The FPU discovery: quick relaxation to a state of apparent equilibrium, which doesn't correspond to energy equipartition, at low energies. The figure is an analogue of the last figure of the FPU paper. The time-averaged energies of a selection of modes are reported vs time, in bilogarithmic scale, for an initial condition with only the lowest-frequency mode excited at energy $E=0.25$, with 63 particles. A state of apparent equilibrium without equipartition is attained at a time of order $10^{5}$. Furthermore, the "final energies" turn out to decrease with frequency (although the "mode numbers" are not exhibited in the figure), so that the "final state" is reminiscent of the initial one.

\subsection{The contribution of Zabusky and Kruskal on the in- tegrable approximations}

A first effect of the FPU work was to stimulate the interest of Zabusky and Kruskal (see the review [11], and [12] [13]), who showed how some features of the FPU system are well interepreted in terms of solitons, that show up when the FPU model is approximated by the Korteweg-deVries partial differential equation, which indeed is an integrable Hamiltonian system. This gave rise to a proliferation of works, which originated a new important branch of research about partial differential equations of integrable type. However, in general one has the impression that in such works the original FPU motivation with respect to statistical mechanics disappeared at all, while they should play a relevant role. Indeed, as will be seen, one might conjecture that at low energies the motions have a prevalent chaotic character, whereas the existence of an integrable approximation pointed out by the "soliton group" seems to rather suggest that 
at low energies the motions may not be sufficiently chaotic for guaranteeing the applicability of the Gibbs statistics. In such a way, in the parlance of the previous section, one would eventually recover a sort of continuity with respect to the completely integrable character of the uncoupled case occurring at vanishing energy.

\subsection{Enters the Chirikov group: a discovery and a conjec- ture}

We now recall how Chirikov, who was the first to point out the relevance of the FPU problem for the foundations of physics, envisaged a clever way by which the FPU paradox could be eliminated, so that classical mechanics would be proved to fail.

Many are the works performed by Chirikov and his group (see the review [14] and [15],[16] [17]), the most known one possibly being that of Izrailev and Chirikov [16]. We believe we do not underestimate such works if we summarize (or interpret) them as consisting of a discovery and of a conjecture. The discovery is illustrated through Figure 2. One considers the same kind of initial condition as in Figure 1, the only change being the value of total energy $E$, which now is increased, being $E=10$ rather than $E=0.25$. Notice that the value of the final time is the same as in the previous figure. This is a relevant point because, for what concerns numerical computations, any moment of history has its value for the final time available, which usually increases (and even very much) at later moments. Now, Figure 2 shows that, by raising energy, a qualitative change apparently occurs because, within the same final time of the previous figure, a "final" state of energy equipartition was attained.

We now try to illustrate the meaning many people attributed to the results of the Chirikov group. First of all, one started speaking of an energy threshold $E^{c}$ having the role of a chaoticity (or stochasticity) threshold or of an equipartition threshold, in the sense that there exists $E^{c}$ such that for $E>E^{c}$ one has equipartition (or rather a quick equipartition), whereas for $E<E^{c}$ one doesn't have equipartition. So, in the parlance used before, the existence of a threshold would restore continuity, since the "classical result" of equipartition obtains only for large enough energies.

However, the relevant quantities for statistical mechanics are not extensive ones such as an energy $E^{c}$, but rather the corresponding intensive quantities such as $\varepsilon^{c}=E^{c} / N$. Indeed one is interested in the thermodynamic limit $N \rightarrow \infty$ with nonvanishing values of the specific quantities $E / N$ and $V / N$ where $V$ is the system's volume. Thus the conjecture usually attributed to the Chirikov group was that the specific energy threshold should vanish in the thermodynamic limit,

$$
\varepsilon^{c}=\frac{E^{c}}{N} \rightarrow 0 \quad \text { for } \quad N \rightarrow \infty
$$

This is the very brilliant idea through which discontinuity is recovered in the thermodynamic limit, since in such a limit only chaotic motions would occur. Numerical results of the Chirikov group seemed to support such a conjecture. 


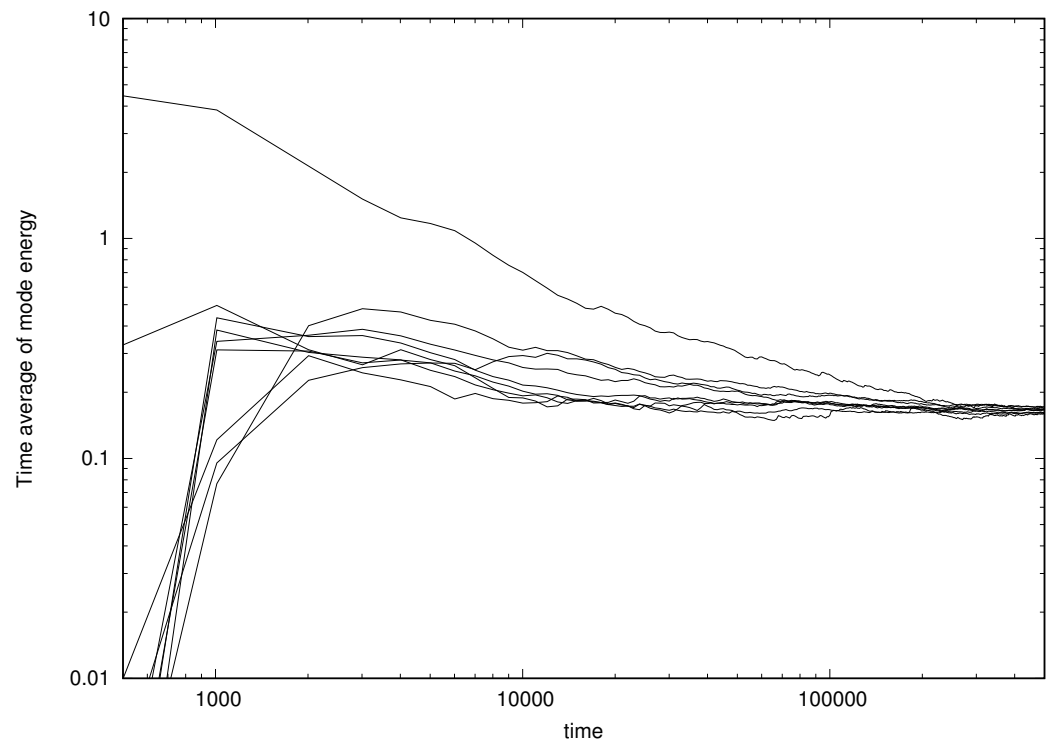

Figure 2: The discovery of the Chirikov group: quick relaxation to a state of apparent equilibrium with apparent energy equipartition, at high enough energies. With the same initial conditions as in Figure 1, but for a higher energy ( $\mathrm{E}=10$ instead of $\mathrm{E}=0.25)$, a state of apparent equilibrium with apparent equipartition is attained within the same time scale.

Notice that in formulating such a conjecture the Chirikov group was following the indications that were available from theory, especially from the Russian school. One should recall that, at those times, the only form of perturbation theory available was the classical one which aims at controlling all points of phase space. The best results obtained up to now, due to Nekhoroshev [18][19], are so formulated that perturbation theory would not be applicable in the thermodynamic limit. Indeed the greatest effort was made later for a variant of the FPU model best suited for applying the theory (see paper [20]), and the result was that the theory can be applied for any $N$, but only for finite values $E$ of energy, i.e., for vanishing specific energy $\varepsilon=E / N$ in the limit $N \rightarrow \infty$. Such a negative result is analogous to a corresponding result holding for KAM theory, which too apparently doesn't apply in the thermodynamic limit. All these negative theoretical results appeared to confirm the conjecture advanced by the Chirikov group.

In this connection we may mention however that when in the year 1974 the senior author, together with Scotti, had two long conversations with Kolmogorov in his apartment at the Moscow University, Kolmogorov explicitly said he was confident that perturbation methods should be applicable in the thermodynamic limit. And actually this was later proven to be the case, as will be recalled below. 
It is conceivable that, through his mastering of probability, he had in mind that the perturbation methods could be extended in a statistical frame (rather than pointwise), which is just the frame relevant for statistical mechanics.

\subsection{The opposite conjecture advanced. A long debate with no clear conclusions}

When the works of the Chirikov group first became known in Milan, a great excitation arose in the group of theoretical physicists, who liked to consider themselves as strictly related, through Caldirola, to Fermi. Soon a check of the Chirikov conjecture was conceived and performed by Bocchieri, Loinger and Scotti [21]. An analogue of the FPU model was considered, which would lend itself to a physical interpretation. The material points were thought of as representing Argon atoms, and the interaction between adjacent atoms was chosen to be the familiar Lennard-Jones one

$$
V(r)=4 V_{0}\left[\left(\frac{\sigma}{r}\right)^{12}-\left(\frac{\sigma}{r}\right)^{6}\right],
$$

which represents a potential well (of depth $V_{0}$ and "size" $\sigma$ ) with a repulsive barrier. However, the most significant result was independent of such details, and just amounted to the fact that the energy threshold in Chirikov's sense was found to be proportional to $N$, at least for $N>10$, through the computations performed for $2 \leq N \leq 100$. So apparently there existed a nonvanishing specific energy threshold $\varepsilon^{C}>0$, more precisely

$$
\varepsilon^{c} \simeq V_{0} / 27
$$

(the senior author has this number $1 / 27$ well fixed in his mind). Soon the possibility of a physical interpretation in connection with Planck's law was advanced (perhaps in some plain way) [22][23] [24], with Cercignani and the senior author entering the game.

A long debate then followed (see the collections of papers in [1] and [2]), especially in Italy, with the aim of deciding which of the two possibilities should hold in the thermodynamic limit, either $\varepsilon^{c} \rightarrow 0$ or $\varepsilon^{c} \rightarrow \varepsilon_{0}>0$. No agreement could however be found, the situation remaining rather confuse, at least for the foundational problem addressed here. Apparently, some new ideas had to be introduced.

\section{Second phase: statistical perturbation theory, relaxation times, role of the integrable ap- proximations, the wide-sense FPU problem}

\subsection{Statistical perturbation theory}

The first progress was of a quite general character, having an impact both for dynamical systems theory and for statistical mechanics. It consists in the proof, 
given in the paper [25], that perturbation theory holds in the thermodynamic limit. A posteriori the main idea underlying such a result might appear quite natural, and really one may wonder how it could happen that it was not found before. But the senior author remembers very well how, together with Benettin and Giorgilli, many attempts were made to tackle the problem, without being able to find the solution. The a posteriori simple solution consists in renouncing to take a pointwise approach in phase space, i.e., to control all initial data, choosing instead to look at the problem in a probabilistic frame. Making reference to an invariant measure, typically the Gibbs one, one should estimate for example the probability of passing from a set of phase space to another one within a given time, or estimate time correlation functions. And indeed such a natural approach had already been followed for example by Neishtadt [26] in his studies of the probability of capture into resonance, which is of interest in celestial mechanics. Here, however, instead of the $L^{1}$ norm in phase space used by Neishtadt, one makes use of the $L^{2}$ norm, which is the one of interest for statistical mechanics. In any case, the new result had the relevant consequence of eliminating from its roots the incredible idea that had pervaded for a long time the community working in the FPU problem, namely, the idea that for systems with a large number of degrees of freedom, only chaotic motions, and not regular ones, would exist. This is even more incredible if one recalls that it is just through studies of a mathematical character on the very FPU problem that Kruskal, Zabursky, Lax and others, had been led to understand how common are the infinite dimensional dynamical systems having an integrable character.

The statistical methods in perturbation theory were later used in [27], where estimates of Nekhoroshev type were obtained for the rotators model, the same considered, as a paradigmatic example, in [25], where only a finite number of perturbation steps had been performed. Results of such a type are now very common, and were obtained for FPU-type models too [28][29][30], and in particular (see [31]) for the variant previously investigated, in which the traditional pointwise methods had been shown not to be applicable in the thermodynamic limit.

In any case, little by little the scientific community came to agree that in the FPU problem the relevant phenomena should be described in terms of specific quantities such as the specific energy $\varepsilon=E / N$, which are the interesting ones in the thermodynaimc limit. Moreover, the quantities should be investigated in statistical terms, typically through time-correlation functions, which are defined by performing averages over initial data distributed according to a Gibbs diestribution. Typical of such an approach is the paper [32].

\subsection{Relaxation times, and attainment of equipartition}

In the meantime a new phenomenon was discovered. We have already recalled how the original FPU result, namely the relaxation to an apparent equilibrium state without equipartition at low energy, had been complemented by the Chirikov's group discovery that equipartition is quickly attained for high enough energies. Now, the further result was that equipartition is attained even at low 


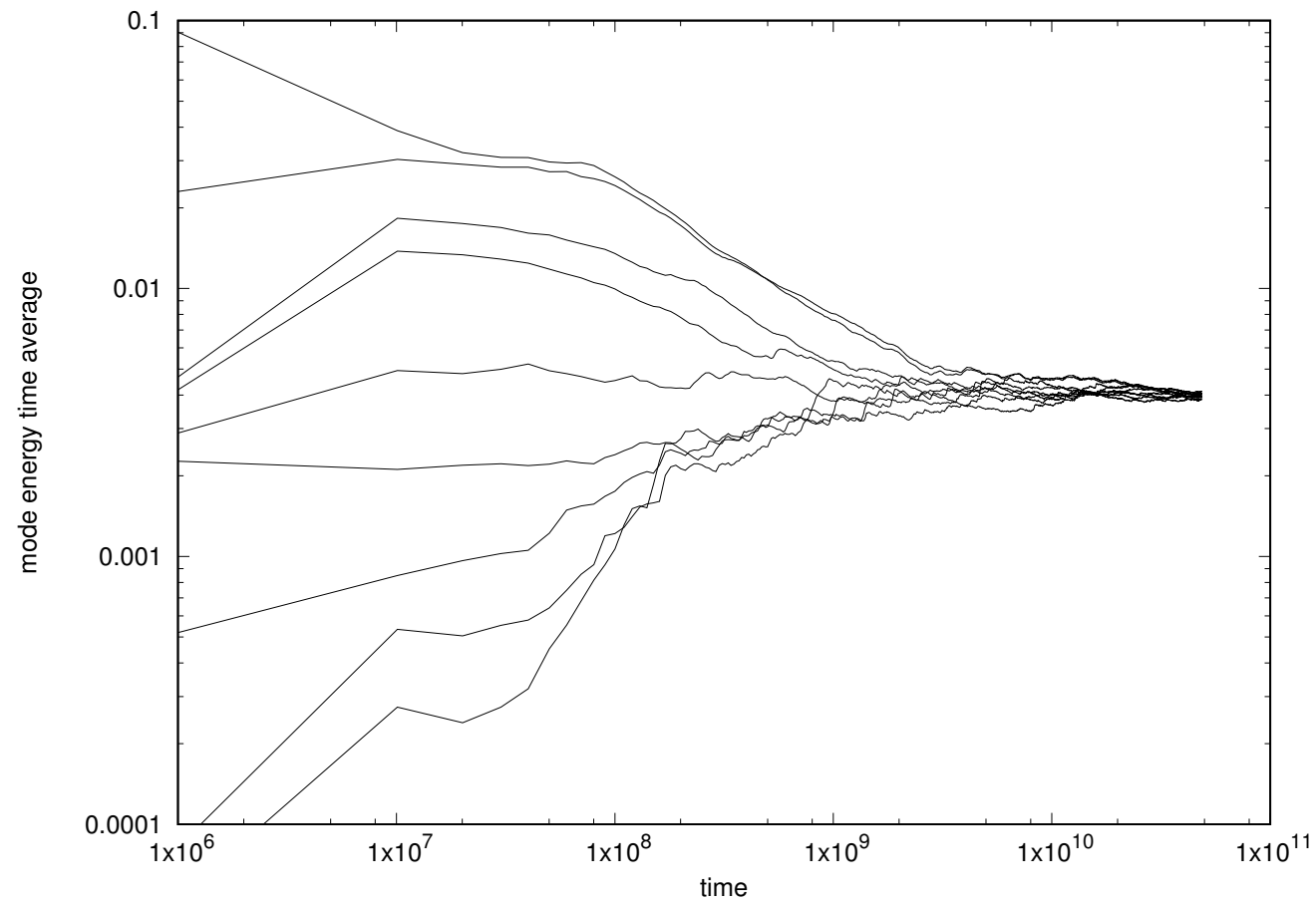

Figure 3: A more recent discovery, i.e., delayed relaxation at low energies. In the same conditions of Figure 1, but for a longer time, a state of apparent equilibrium with apparent equipartition is eventually attained (here, at a time of order $\left.10^{11}\right)$.

energies, after a suitably long relaxation time. Apparently this was first observed in the paper [33] through a figure (Figure 9) exhibited in that paper, a figure that in the Milan group is usually called, in a colloquial way, the Giorgilli shower. Later, a large evidence was given in works performed in Padua around Benettin and Ponno [34] [35], which provided extremely detailed numerical estimates of the relaxation times involved. See also [36], and [37]. In fact, below a certain specific energy threshold, somehow reminiscent of the Chirikov threshold, two time scales are met. At first, energy is found to spread, forming a little packet around the modes initially excited, and this defines the first time scale, which is actually the one observed in the original FPU paper. Then on a second time scale equipartition is attained. Here we illustrate the phenomenon of the two time scales through Figure 3. This is just a prolongation of Figure 1 to longer times, and one sees that, after the FPU state of apparent equilibrium without equipartition, which had been observed at times of the order $10^{6}$, equipartition 
is eventually attained at a later time ${ }^{2}$ of the order $10^{11}$. The computations performed in Padua show that the time required for attaining equipartition grows as an inverse power of $\epsilon$. In fact, no complete agreement is found here, because it cannot be excluded that the power depends on the range of $\varepsilon$. This would indeed be the familiar Nekhoroshev phenomenon, that eventually would lead to an exponential dependence on $1 / \varepsilon$.

\subsection{Role of the integrable approximations}

The next contribution concerns the role the integrable approximations play for the FPU problem. In recent times this point was discussed at length by the Padua group. Here one meets with a quite general problem, first explicitly pointed out by Siegel in the year 1941 [38]. If one considers a definite model, say the FPU one, defined through a certain Hamiltonian, then the dynamical system is represented by a point in a suitable function space, and one may ask how much does it differ from other "nearby" points. One then finds that arbitrarily near to the given point there exist both integrable and non integrable systems. In particular one sees that the particular integrable nearby system considered by FPU really is an exceptional one, in reason of its linearity. Many other integrable systems perhaps exist, which constitute much better approximations to the FPU model. This had in fact been the starting point of the old works of Kruskal, Zabusky and others. Subsequently this point of view was reconsidered in an important paper in which Ferguson, Flashka and McLaughlin [39] showed that a Toda model indeed makes a better job. Actually, for any FPU model one should choose the corresponding "tangent Toda model", whose parameters are suitably chosen as to best approximate the given FPU model. A review of the problem of the integrable approximations can be found in [40].

Several studies were performed concerning such a tangent Toda model. A first result consists in explaining what occurs in the first time scale. In the very words of Benettin and Ponno, it turns out that "the formation of a packet reminiscent of the initial state is an integrable phenomenon". Namely, the attainment of the apparent equilibrium observed in the original FPU paper is perfectly well reproduced by the tangent Toda model, and has nothing to do with chaoticity. Moreover, the dynamics of the tangent Toda lattice also explains very well the "statistical" phenomena occurring for generic initial data and described through time-correlation functions, which were first observed, in an FPU frame, in the paper [32].

\subsection{Necessity of a generalized wide-sense FPU problem}

Thus, finally, in the scientific community an agreement was found on the fact that, starting from exceptional initial data with a few modes excited, equipar-

\footnotetext{
${ }^{2}$ Here, as in the previous figures, the computed time averages are not accurate for times that are small with respect to the final one. In fact, they are computed using the instantaneous values at times multiples of a step depending on the final time. This explains why the curves of Figure 3 do not superpose with those of Figure 1 at corresponding times.
} 
tition is eventually attained after a sufficiently long time. Moreover, this occurs even in the thermodynamic limit, which involves energy per particle $\varepsilon=E / N$ rather than just energy $E$. So, the "little discovery" of FPU was apparently vanified: the relaxation to a nonequipartition state observed by FPU was just an apparent one, and after a certain amount of time a "final" relaxation to a state of equipartition occurs. In such a way the FPU paradox disappears, and classical mechanics is apparently proven to fail, as we all were thought "since always".

Things however are not that easy. In order to use classical Gibbs statistics in computing the averages, more than mere equipartition is needed, as particularly stressed in several works of the Kozlov school that will be shortly discussed in the next subsection. In the same vein, in the frame of numerical works it was found that, after equipartition is attained, one meets with some sort of intermittency, in which the system jumps between states close to regular orbits. This is shown in Figure 6 of the paper [41] by Giorgilli, Paleari and Penati. Another example is the statistics of the return times (see Figure 3 of the paper [42] by Danieli, Campbell and Flach), which seems to be different from that expected according to Gibbs measure. See aalso the paper [43] on the Gross-Pitaevskii model. ${ }^{3}$

This is the reason why the FPU problem should be more appropriately formulated in a wider sense with respect to the original one. Instead of checking whether equipartition is attained starting from exceptional initial data, one should investigate whether, starting from generic initial data extracted according to a Gibbs distribution, the standard methods of equilibrium statistical mechanics can be applied (as was made for example in the paper [32]). This is what might be called the wide-sense FPU problem. Concerning the possible answers to the problem thus formulated, our expectation should by now be evident. Namely: the great impression produced on the scientific community by the soliton phenomena discovered since the year 1965 by Zabusky and Kruskal, and their direct impact on the FPU model pointed out by Ferguson, Flashka and McLaughlin [39] and particularly exhibited by the Padua group, constitute such an enormous barrier against the naive conception that chaotic motions should always prevail, that the validity of the Gibbs statistics at low energies seems to us to be at least extremely improbable.

One can finally ask which quantities of physical interest should be investigated in order to exhibit that the Gibbs statistics is not justified at low energies, after energy equipartition has been attained. This is far from trivial, because, after all, in the FPU problem everything started from the fact that it is indeed energy equipartition that is apparently involved when one refers to the fundamental experiments that led to the introduction of quantum mechanics. The problem actually is a very difficult one. Perhaps a solution may be found by considering that in such questions the main role is not played by the energy possessed by a system, but by the energy it can exchange with an apparatus, typically with a calorimeter in the case of a specific heat measurement. See

\footnotetext{
${ }^{3}$ We thank Simone Paleari for pointing out to us the existence of the two last works mentioned, just a few days before the completion of the present paper.
} 
for example [44] [45]. One might very well have energy equipartition and at the same time negligible energy exchanges for some degrees of freedom. This was indeed repeatedly pointed out by Boltzmann himself in connection with the specific heat difficuties, making reference to his preferred example, i.e., that of a perfectly smooth sphere, which can possess an arbitrarily large rotational energy, without exchanging it at all. Interesting indications in this connection were given some time ago [46] for the energy exchanges of an oscillator with a gas. Moreover, an important role may be played by long-range electric interactions, as shown by the results illustrated in the next section, which concern theoretical estimates of the spectral lines for a realistic FPU-like model of an ionic crystal.

\subsection{Recent studies on the validity of the Gibbs statistics}

It was recalled above how the problem of finding invariant measures in systems near to integrable ones is not simple at all. First of all, as pointed out by Kozlov ([47], see also [48]) through a counterexample, energy equipartition does not imply ergodicity: in the case of weakly coupled oscillators of the same frequency, energy equipartition is attained, but the system is not ergodic because it admits further integrals of motion in addition to total energy. Furthermore it was pointed out (see [49], and the paper [50] with Treshchev) that ergodicy is not an useful property in order to describe the approach to equilibrium. Rather, the approach to equilibrium should be described by requiring that the phase average of any observable converges, as $t \rightarrow \infty$, to a well defined value, namely, in mathematical terms, requiring that all solutions of the Liouville equations converge in weak sense to a well defined measure. Then such a limit measure turns out to be the equilibrium one, and indeed is equal to the time-average of the initial measure. However, the measure thus found coincides with the Gibbs one only if further conditions are satisfied, and it is not clear whether this occurs in the FPU case.

Moreover, one also meets with the following problem: if one computes the phase averages through a measure different from the Gibbs one, do the thus found macroscopic quantities satisfy the principles of thermodynamics? For example, does the exchanged heat have an integrating factor? Or, is the second principle satisfied? Now, in the work [51] by Ponno it was proved that the latter requirement restricts the choice of the admissible families of measures to the so called Tsallis distributions [52], which contain the Gibbs measure as a particular case. Moreover, in the work [53] by one of the present authors, it was shown that the exchanged heat can be proven to admit an integrating factor, even if one limits oneself to consider time averages, without passing to consider phase averages.

All these remarks indicate that the problem of obtaining thermodynamics from mechanics is rather complex and not yet settled. As an aside comment, we point out that problems of an analogous type are also met in the theory of glasses. This problem was addressed, just in connection with the FPU problem, in an old paper by Fucito et al. [54] of the year 1982, to which much attention 

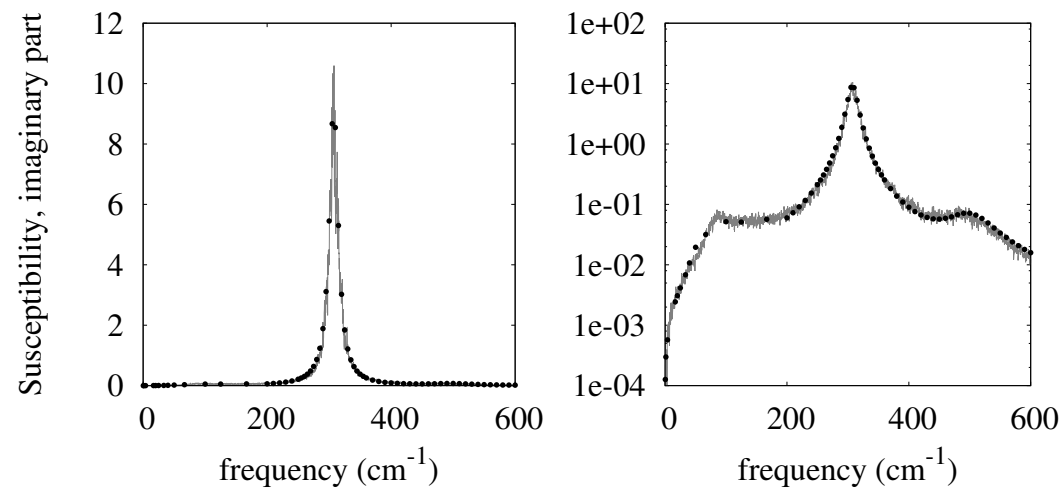

Figure 4: Spectral line for a LiF crystal at room temperature, through the curve of susceptibility (imaginary part) vs frequency: computed curve (solid line), experimental data (circles). Left panel in linear scale, right panel in semilogarithmic scale. Experimental data from Palik E., Handbook of optical constants of solids (Academic Press, Amsterdam, 1998).

was paid in the review [7].

\section{The realistic FPU-like model of an ionic crys- tal}

There are phenomena that are usually considered as typically quantum ones, at the point of appearing perfectly unintelligible from a classical standpoint (in Born's words). The most characteristic one is the existence of sharp spectral lines which, in the Bohr familiar picture, seem to require the existence of energy levels, i.e., of quantization. This is not true, as is shown by Fig. 4 which reports, versus frequency $\omega$, the imaginary part of susceptibility $\chi$ (i.e., essentially, the absorption coefficient), for a Lithium Fluoride (LIF) crystal $^{4}$. The circles are the experimental data, and the continuous line is the corresponding theoretical spectral curve. Such a theoretical curve, as also the two further ones reported below, were previously reported in the papers [55] and [56]. ${ }^{5}$

The relevant point is that the theoretical curve of Fig. 4 was obtained through a completely classical molecular dynamics (or MD) computation, solv-

\footnotetext{
${ }^{4}$ Lithium Fluoride is considered to possibly be the simplest crystal, playing for crystals a role analogous to that of Hydrogen for gases.

${ }^{5}$ Actually, the figures are not quite the same, and there is a small difference. Because the value of the parameter $\chi^{\infty}$ - see below - was chosen here with the value 0.147 , rather than with the value 0.153 chosen in [56]. Anyway, one can see that such a difference produces essentialy no observable change in the corresponding figures, which are thus robust enough to variations of the parameters, at least in sufficiently small ranges.
} 
ing the Newton equations for a model in which the ions $\mathrm{Li}^{+}$and $\mathrm{F}^{-}$are dealt with as point particles subject to two-body forces, as in a kind of realistic FPUlike model. In our opinion, this figure is the most striking example intimating (we are mimicking here the very words of Fermi) that the relations between classical and quantum mechanics should be reconsidered under some new light.

In order to understand the figure, one should first of al recall that the spectra are described by lines only in a first rough approximation, and that one should rather refer to spectral curves, which report spectral features, first of all susceptibility $\chi$ versus frequency $\omega$, and then refractive index $n$, reflectivity $R$ and so on, which are all expressed in terms of susceptibility.

A posteriori, the procedure to be followed in order to obtain the spectrum of Fig. 4 in a classical frame can be described in a plain and simple way, which may even appear obvious, and will be illustrated in a moment. However, such a description of the procedure doesn't do justice to the true situation. Indeed, the production of the figure actually required from us to cover a long path and to overcome hard conceptual difficulties. Eventually, the path became possible only after a full appreciation of the contributions to general features of classical elecrodynamics that were given by Dirac in the year 1938 and by Wheeler and Feynman in the year 1945. An illustration of such very interesting points would lead us too far from the main theme of the present review.

The simple-minded way of dealing with the problem of the spectral curves, which allows one to eliminate the necessity of quantization, is to take into account the existence of the Green-Kubo linear response theory, which became available only after the late years fifties. Already in the years twenties an analogous step was accomplished by Schrödinger, albeit in the case of single atoms with their energy levels, and the intensity of the lines was then estimated through the corresponding transition probabilities induced by an incoming electromagnetic wave, acting as an external perturbation. The Green-Kubo approach, instead, develops within a statistical mechanical frame. In such a case any reference to energy levels is eliminated, because the macroscopic response is expressed through polarization, which is defined microscopically in terms of the positions $\mathbf{x}_{j}$ of the ions (of charge $e_{j}$ ) as

$$
\mathbf{P}(t)=\frac{1}{V} \sum_{j} e_{j} \mathbf{x}_{j}(t),
$$

$V$ being the value of the considered cell (see below), and this makes perfect sense also in purely classical terms. Finally, the susceptibility curve $\chi(\omega)$ turns out to be defined essentially as the Fourier transform of the time-correlation of polarization. More precisely one has to consider the corresponding tensor quantities, and one has

$$
\chi_{i j}(\omega)=\beta V \int_{0}^{+\infty} e^{-i \omega t}\left\langle P_{i}(t) \dot{P}_{j}(0)\right\rangle d t .
$$

with a statistical average $\langle\cdot\rangle$ over the initial data, and $\beta=1 / k_{B} T$. In the case of $\mathrm{LiF}$ one meets with an isotropic case, and one has $\chi=\sum \chi_{i i} / 3$. 
In this way Fig. 4 was obtained, replacing the mean over the initial data, with the mean over time averages along a small number of trajectories computed by numerical simulations. The model considered is a standard one in solid state physics: we consider a system of point particles describing the two species of ions, in a cell containing 4096 particles, with periodic boundary conditions. The interactions are the Coulomb ones (cared for their long range feature through a standard Ewald procedure), plus a two-body short range "effective force", which is introduced in a phenomenological way. This is done, following a procedure first introduced by Born in order to implicitly take into account the role of the electrons, whose degrees of freedom don't show up in the model. In the same phenomenological way one also introduces "effective charges" which are substituted for the true charges of the ions in the expression of the mutual Coulomb forces. Finallly, the contribution of the electrons to the permittivity $\varepsilon_{i j}(\omega)=\delta_{i j}+4 \pi \chi_{i j}(\omega)$ is taken into account through a constant $\chi^{\infty}$.

Thus, the model is a "first-principle" one only for what concerns the ions, whereas the electrons are dealt with in a phenomenological way. Treatments of such a type turn out to be sufficient in order to deal with the infrared spectra of ionic crystals, as are usually employed also in quantum models. Now, the phenomenological way used for dealing with the electrons is certainly a drawback of the model, but in our opinion it is irrelevant for the main point we are making here, namely, that energy quantization is not at all necessary for a theoretical calculation of the infrared spectra of ionic crystals. In other words one might say: the decree "no energy levels, no lines" is not correct.

For more details, we refer the reader to the paper [56]. We finally come to a feature that came to light in such a paper, and has a direct impact on the general problem under discussion here, which concerns the validity of the Gibbs statistics for FPU-like models at low temperatures.

The aim of the paper [56] was to determine how the agreement between theoretical and experimental spectra, already checked in [55] to be very good at room temperature, may depend on temperature. This led us to bump into the general problem of validity of the Gibbs statistics, and the relevant feature turned out to be the procedure to be used for identifying temperature in mechanical terms. In the previous paper [55] the identification used was the standard one of Molecular Dynamics in terms of kinetic energy $K$, that follows from Gibbs statistics, namely,

$$
<K>=\frac{3}{2} N k_{B} T,
$$

the average being performed with respect to Gibbs measure at temperature $T$.

In the new paper [56] it was found that such a standard identification produces a good agreement at room temperature and at higher ones, actually $605 \mathrm{~K}$, $840 \mathrm{~K}$ and $1060 \mathrm{~K}$ (just below melting), while a disagreement was observed at the two lower temperatures considered, namely, $85 \mathrm{~K}$ and $7.5 \mathrm{~K}$. This is shown by Fig. 5, where the reflectivity curves are reported for the six temperatures, and the agreement is seen to be partially lost at $85 \mathrm{~K}$, and even more at $7.5 \mathrm{~K}$, where the computed reflectivity sticks to the value 1 . However, it occurred to us 


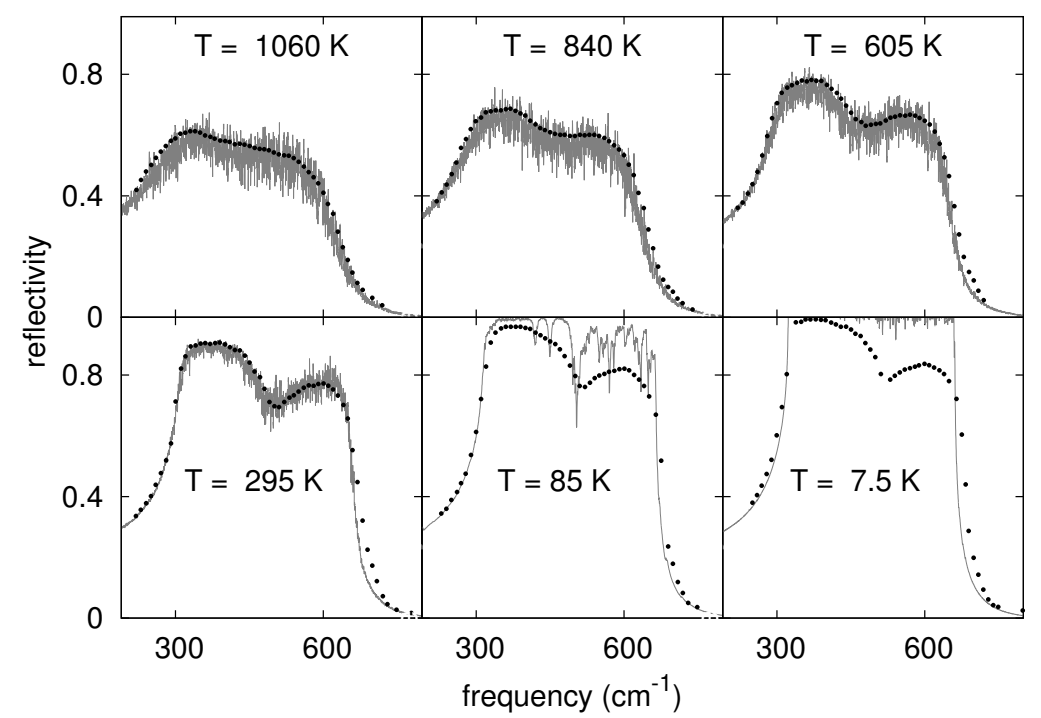

Figure 5: Reflectivity of LiF as a function of frequency at six temperatures. Results from calculations (solid line) are compared with experimental data (points) taken from J. R. Jasperse, A. Kahan, J. N. Plendl, S. S. Mitra, Phys. Rev. 146 (1966) 526.

to find that a pretty good agreement is recovered at the two low temperatures too, if in the computations one inserts "by hand" suitable "elevated temperatures", namely $180 \mathrm{~K}$ and $125 \mathrm{~K}$ respectively, as if one were somehow taking the existence of a "zero-point energy" into account. ${ }^{6}$ This is shown in Fig. 6.

Irrespective of such a possible interpretation, it is a fact that agreement is recovered if one renounces to stick at the Gibbs prescription, and thus to the corresponding standard identification of temperature in terms of kinetic energy. In other words, at low temperatures classical mechanics still applies and reproduces well the spectral curves, if one admits that Gibbs' statistical mechanics doesn't.

\section{Conclusions}

We can now come back to the main theme discussed in the present review, namely, whether the FPU paradox, intimating that the relations between clas-

\footnotetext{
${ }^{6}$ Work is in progess in order to clarify this point.
} 


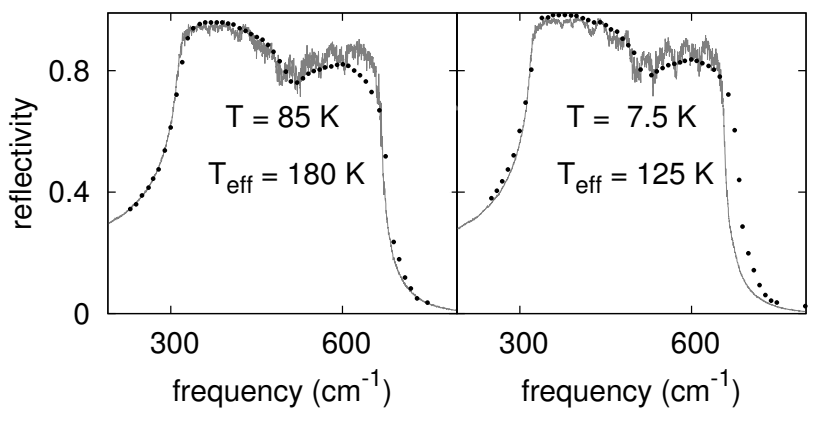

Figure 6: Same as Fig. 5, for the two experimental temperatures $85 \mathrm{~K}$ and $7.5 \mathrm{~K}$, where calculations were performed at the effective temperatures of $180 \mathrm{~K}$ and $125 \mathrm{~K}$, respectively.

sical and quantum mechanics should be reconsidered under some new light, is still alive. We saw how, after having been apparently vanified by the discovery that energy equipartition is eventually attained, the paradox reappeared in connection with a more general problem, namely, whether Gibbs statistics is justified at low temperatures. We illustrated how several indications exist, in the FPU literature and in works of the Kozlov school, that this may not be the case. Then we saw how this fact is strongly supported by new numerical results on an FPU-like realistic model of an ionic crystal, which reproduce in an impressively good way the infrared spectral curves, a phenomenon usually considered so characteristic of quantum physics as to be even unconceivable in a classical frame.

We finally add a free comment on the problem of the relations between classical and quantum mechanics. The point we make is that such relations should be conceived in a broader context, namely as relations between classical physics and quantum physics. Indeed, as was particularly stressed by Feynman in his Nobel lecture, the phenomena where classical mechanics was judged to fail and quantum mechanics emerged, actually are all phenomena of atomic physics, which involve in an inextricable way particles and electromagnetic field. However, the classical theory of the electromagnetic field is much broader than might be guessed from standard textbooks. For example, the ions considered in the model just illustrated should come to rest, due to the radiation they would emit according to the Larmor formula. But this is not the case for a macroscopic system, as was shown by Wheeler and Feynman. These are very interesting problems, which, however, we couldn't address in the present review. 


\section{References}

[1] Campbell, D.K., Rosenau, P. and Zaslavsky, G.M, The FPU problem - the first fifty years, Chaos, 2005, Vol. 15, pp. 015101.

[2] Gallavotti, G., (ed), The Fermi-Pasta-Ulam Problem: A Status Report, Lecture Notes in Physics, Vol. 728, Springer Verlag (Berlin, 2007).

[3] Schilpp, P.A., Albert Einstein: philosopher-scientist, Tutor (New York, 1951).

[4] Carati, A., Galgani, L., Progress along the lines of the Einstein Classical Program: An enquiry on the necessity of quantization in light of the modern theory of dynamical systems. Notes (in an extremely preliminary form) for a course on the Foundations of Physics at the Milan University, available in Italian at the home pages of the authors.

[5] Born, M., Atomic physics, Dover (New York, 1969)

[6] Carati, A., Galgani, L., Giorgilli, A., The Fermi-Pasta-Ulam problem as a challenge for the foundations of phyics, Chaos, 2005, Vol. 15, pp. 015105.

[7] G. Benettin, A. Carati, L. Galgani and A. Giorgilli: The Fermi-Pasta-Ulam problem and the metastability perspective, in ref. [2], pp. 151-190.

[8] H. Poincaré, Les méthodes nouvelles de la mécanique céleste, Vol. I, Chapter 5 .

[9] Fermi, E, Pasta, J. and Ulam, S., Studies of nonlinear systems, in Fermi, E., Collected Papers, Roma: Accademia Nazionale dei Lincei, 1965, Vol.II, N. 266, pp. 978-993.

[10] Fermi, E., Beweis, dass ein mechanisches Normalsystem in Algemeinen quasi-ergodisch ist, reprinted in Collected Papers, N. 11a, Vol I, pp 79-86.

[11] Zabusky, N.J., Fermi-Pasta-Ulam, solitons and the fabric of nonlinear and computational science. History, sinergetics, and visiometrics Chaos, 2005, pp. 015102.

[12] Zabusky, N.J, Kruskal, M.D., Interaction of solitons in a collisionless plasma and the recurrence of initial states, Phys. Rev. Lett, 1965, 15, pp. 240-243.

[13] Gardner, C.S., Greene, J.M., Kruskal, M.D., Miura, R.M., Method for solving the Korteweg-de Vries equation, 1967, Phys. Rev. Lett., Vol. 19, pp. 1095-1097.

[14] Berman, G.P., Izrailev. F.M., The Fermi-Pasta-Ulam problem: Fifty years of progress, 2005, Chaos, Vol. 15, pp. 105104.

[15] Chirikov, B.V., J. Nucl. Energy: Part C, 1960, Vol. 1, 253. 
[16] Izrailev, F.M. and Chirikov, B.V., Statistical properties of a nonlinear string, Sov. Phys. Dokl., 1966, Vol. 11, pp. 30-34.

[17] Izrailev, F.M. Khisamutdinv, A.I., and Chirikov, B.V., Numerical experiments with a chain of coupled anharmonic oscillators, 1968, Report 252, Institute of Nuclear Physics, Novosibirsk USRR (English translation LA4440-TR, Los Alamos 1970).

[18] Nekhoroshev, N.N., An exponential estimate of the time of stability of nearly-integrable Hamiltonian systems, Russ. Math. Surv., 1977, Vol. 32, pp. 1-65.

[19] Benettin, G., Galgani, L. and Giorgilli, A., A proof of Nekhoroshev's theorem for the stability times in nearly integrable Hamiltonian systems, $\mathrm{Ce}$ lestial Mech., 1985, Vol. 37, pp. 1-25.

[20] Bambusi, D.D. and Giorgilli, A., Exponential stability of states close to resonance in infinite dimensional Hamiltonian systems, Jour. Stat. Phys., 1993, Vol, 71 p. 569.

[21] Bocchieri, P., Scotti, A., Bearzi, B. and Loinger, A., Anharmonic chain with LennardJones interaction, Phys. Rev. A, 1970, Vol. 2, pp. 2013-2019.

[22] Galgani, L. and Scotti, A., Planck-like distribution in classical nonlinear mechanics, Phys. Rev. Lett., 1972, Vol. 28, pp. 1173-1176.

[23] Cercignani, C., Galgani L. and Scotti A., Zero-point energy in classical nonlinear mechanics, Phys. Lett. A, 1972, Vol. 38, pp.403-405.

[24] Galgani, L., and Scotti A., Recent progress in classical nolinear dynamics, Rivista del Nuovo Cimento, 1972, Vol. 2, pp. 189-209.

[25] Carati, A., An averaging theorem for Hamiltonian dynamical systems in the thermodynamic limit, J. Stat. Phys., 2007, Vol. 128, pp. 1057-1077.

[26] Neishstadt A.I., Averaging in multifrequency systems, II, Sov. Phys. Dokl., 1976 , Vol. 21, pp. 80-83.

[27] De Roeck, W. and Huveneers, F., Asymptotic localization of energy in nondisordered oscillator chains, Comm. Pure Appl. Math., 2015, Vol. 68, pp. $1532-1568$.

[28] Maiocchi, A., Bambusi, D. and Carati, A., An averaging theorem for FPU in the thermodynamic limit, J. Stat. Phys., 2014, Vol. 155, pp. 300-322.

[29] Carati, A. and Maiocchi, A., Exponentially long stability times for a nonlinear lattice in the thermodynamic limit, Comm. Math. Phys., 2012, Vol. 314, pp. 129-161. 
[30] Giorgilli, A., Paleari, S. and Penati T., An extensive adiabatic invariant for the Klein-Gordon model in the thermodynamic limit, Annales Henri Poincaré, 2015, Vol. 16, pp. 897-959.

[31] Maiocchi, A., Freezing of the optical-branch energy in a diatomic FPU chain, preprint.

[32] Carati, A., Galgani, L., Giorgilli, A. and Paleari, S., FPU phenomenon for generic initial data, Phys. Rev. E, 2007, Vol. 76, pp. 022104.

[33] Berchialla, L., Galgani, L. and Giorgilli, A., Localization of energy in FPU chains, Discrete and continuous dynamical systems A, 2004, Vol. 11, pp. $855-866$.

[34] Benettin, G. and Ponno, A., Time-scales to equipartition in the FPU problem: Finite-size effects and thermodynamic limit, J. Stat. Phys., 2011, Vol. 144, pp. 793-812.

[35] Benettin, G., H. Christodoulidi, H. and Ponno, A., The Fermi-Pasta-Ulam problem and its underlying integrable dynamics, J. Stat. Phys., 2013, Vol. 152, pp. 195-212.

[36] Berchialla, L., Giorgilli, A. and Paleari, S., Exponentially long times to equipartition in the thermodynamic limit, Physics letters A, 2004, pp. 267172.

[37] Paleari, S. and Penati, T., Equipartition times in a FPU system, Discrete and continuous dynamical systems, 2005, Supplementary Vol. pp. 710-719.

[38] Siegel, C., On the integrals of canonical syatems, Annals of Mathematics. 1941, Vol. 42, pp. 806-822.

[39] Ferguson, E.E, Flashka, H. and McLaughlin D.W., Nonlinear Toda modes for the Toda chain, J. Comp. Physics, 1982, Vol. 45, pp. 157-209.

[40] Bob Rink, An integrable approximation for FPU lattice, in ref. [2], pp. 283-301.

[41] Giorgilli, A., Paleari, S. and Penati, T., Local chaotic behaviour in the FPU system, DCDS-B, 2005, Vol. 4, pp. 991-1004.

[42] Danieli, C., Campbell, D.K. and Flach S., Intermittent FPU dynamics at equilibrium, Phys. Rev. E, 2017, Vol. 95, pp, 060202.

[43] Mithun, T., Kati, Y., Danieli, C. and Flach, S., Weakly nonergodic dynamics in the Gross-Pitaevskii lattice, Phys. Rev. Lett., 2018, Vol. 120, pp. 184101.

[44] Carati, A,. Galgani, L., On the specific heat of the Fermi-Pasta-Ulam systems, J. Stat. Phys., 1999, Vol, 94, pp. 859-869. 
[45] Carati, A,. Galgani, L., Metastability in specific heat measurements: simulations with the FPU model, Europh. Lett., 2006, Vol. 74 , pp. 528-534.

[46] Carati, A., Galgani, L., Pozzi, B., Levy flights in Landau-Teller model of molecular collision, Phys. Rev. Letts., 2003, Vol. 90, p. 010601.

[47] Kozlov, V.V., Gibbs ensembles, equidistribution of energy of sympathetic oscillators and statistical models of thermostat, Regular and Chaotic Dynamics. 2008, Vol. 13, pp. 141-154.

[48] Kozlov, V.V, Thermal equilibrium by Gibbs and Poincaré, 2002 (Idshevsk, Moscow), in Russian.

[49] Kozlov, V.V., On justification of Gibbs distribution, Regular and Chaotic Dynamics. 2002, Vol. 7, pp. 1-10.

[50] Kozlov, V.V., Treshchev, D.V., Weak convergence of solutions of Liouville equation for non linear Hamiltonian systems, Theor. and Math. Phys., 2003, Vol. 134, pp. 339-350.

[51] Ponno, A., A theorem on the equilibrium thermodynamics of Hamiltonian systems, Physica A, 2006, Vol. 359, pp. 162-176.

[52] Tsallis, C., Possible generalization of the Boltzmann-Gibbs statisitcs, J. Stat. Phys., 1998, Vol. 52, 479-487.

[53] Carati, A., On the definition of temperature using time-averages, Physica A, 2006, Vol. 369, pp. 417-431.

[54] Fucito, E., Marchesoni, F., Marinari, E., Parisi, G., Peliti, L., Ruffo, S, and Vulpiani, A., Approach to equilibrium in a chain of nonlinear oscillators, J. Phys., 1982, Vol. 43, pp. 707713.

[55] Gangemi, F., Carati, A., Galgani, L., Gangemi, R. and Maiocchi, A., Agreement of classical Kubo theory with the infrared dispersion curves $n(\omega)$ of ionic crystals, Europhys. Lett., 2015, Vol. 110, pp. 47003.

[56] Carati, A., Galgani, L., Maiocchi, A., Gangemi, F. and Gangemi, R., Classical infrared spectra of ionic crystals and their relevance for statistical mechanics, Physica A, 2018, Vol. 506, pp. 1-10. 